\title{
Relation between stroke severity, patient characteristics and CT-perfusion derived blood-brain barrier permeability measurements in acute ischemic stroke
}

\author{
Alexander D. Horsch · Jan Willem Dankbaar - Tom van Seeters · Joris M. Niesten • \\ Merel J.A. Luitse · Pieter C. Vos · Irene C. van der Schaaf · Geert-Jan Biessels · \\ Yolanda van der Graaf · L. Jaap Kappelle · Willem P.Th.M. Mali · Birgitta K. Velthuis
}

Received: 13 August 2014 / Accepted: 22 January 2015 / Published online: 27 February 2015

(C) The Author(s) 2015. This article is published with open access at Springerlink.com

\begin{abstract}
Purpose Increased blood-brain barrier permeability (BBBP) can result from ischemia. In this study the relation between stroke severity, patient characteristics and admission BBBP values measured with CT-perfusion (CTP) was investigated in acute ischemic stroke patients.

Methods From prospective data of the Dutch Acute Stroke Study 149 patients with a middle cerebral artery stroke and extended CTP were selected. BBBP values were measured in the penumbra and infarct core as defined by CTP thresholds, and in the contra-lateral hemisphere. The relation between stroke (severity) variables and patient characteristics, including early CT signs, dense vessel sign (DVS), time to scan and National Institute of Health Stroke Score (NIHSS), and BBBP parameters in penumbra and infarct core was quantified with regression analysis.
\end{abstract}

A. D. Horsch, MD, MRCS $(\bowtie) \cdot$ J. W. Dankbaar

T. van Seeters · J. M. Niesten · P. C. Vos · I. C. van der Schaaf

W. P.Th.M. Mali · B. K. Velthuis

Department of Radiology, University Medical Center Utrecht,

Heidelberglaan 100, HP E01.132,

3584 CX, Utrecht, The Netherlands

e-mail: alexanderhorsch@gmail.com

M. J.A. Luitse · G.-J. Biessels · L. J. Kappelle Department of Neurology, Utrecht Stroke Center, University Medical Center Utrecht,

The Netherlands

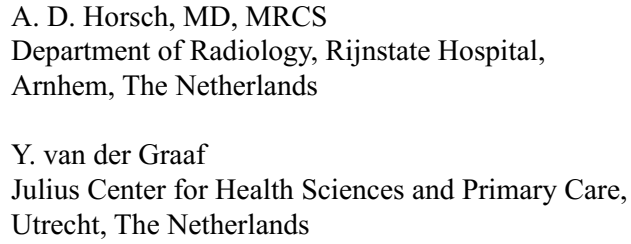

Results Early CT signs were related to higher BBBP values in the infarct core $(B=0.710)$, higher ipsi- to contra-lateral $\mathrm{BBBP}$ ratios $(\mathrm{B}=0.326)$ and higher extraction ratios in the infarct core $(B=16.938)$. Females were found to have lower BBBP values in penumbra and infarct core $(B=-0.446$ and -0.776 respectively) and lower extraction ratios in the infarct core $(B=-10.463)$. If a DVS was present the ipsi- to contra-lateral BBBP ratios were lower $(B=-0.304)$. There was no relation between NIHSS or time to scan and BBBP values.

Conclusion Early CT signs are related to higher BBBP values in the infarct core, suggesting that only severe ischemic damage alters BBBP within the first hours after symptom onset.

Keywords Acute ischemic stroke - Early CT signs · Dense vessel sign - CT perfusion · Blood-brain barrier permeability

\section{Introduction}

Acute ischemic stroke induces damage to the blood-brain barrier (BBB) [1]. Alterations in the BBB integrity related to stroke severity, duration of ischemia and reperfusion have been associated with development of hemorrhagic transformation (HT) [1-3]. BBB permeability (BBBP) can be calculated from CT perfusion (CTP) data by obtaining an extended CTP acquisition and measuring progressive leakage of iodinated contrast out of the cerebral vessels [4]. As currently many stroke clinics routinely obtain CTP on admission in stroke patients, BBBP measurements with CTP could be a practical addition in prognostic and diagnostic stroke imaging [5]. To better understand the concept 
of permeability, thorough evaluation of BBBP measurements with CTP in stroke patients is required.

On arrival to the emergency department, the severity of stroke and ischemic damage differs considerably between patients. Since ischemia is thought to alter BBB integrity, BBBP is likely to be related to stroke severity at the time of CT scanning at admission. Furthermore, several factors intrinsic to the patient have been associated with increased BBBP. These factors include age, elevated diastolic pressure, increased glucose levels and atrial fibrillation [3, 6-8]. If BBBP measurements are an indicator of ischemic damage to the BBB there should be a relationship between these factors and CTP BBBP measurements at time of admission.

The purpose of this study was to evaluate the relationship between stroke severity, patient characteristics, and CTP BBB permeability values in a consecutive prospectively collected series of acute ischemic middle cerebral artery (MCA) stroke patients.

\section{Materials and methods}

\section{Study design}

In this study we used data from the Dutch Acute Stroke Study (DUST). This is a large multicenter cohort study which aims to assess the additional value of CTP and CT angiography (CTA) in predicting outcome of acute ischemic stroke patients. Prospective inclusion was done between May 2009 and August 2013, and patients were enrolled in 14 DUST hospitals [9].

Inclusion criteria for this study were: age $\geq 18$ years, suspected ischemic stroke of less then $9 \mathrm{~h}$ duration and National Institute of Health Stroke Scale (NIHSS) score of $\geq 2$ (or 1 if an indication for thrombolysis was present). Exclusion criteria were known renal failure or contraindications to iodinated contrast material. This study was approved by the central ethics committee in the UMC Utrecht as well as the local institutional ethical review boards of the participating centers. All patients or family gave signed informed consent, unless a patient died before consent could be obtained. The need for consent in that case was waived by the medical ethics committee [9]. From this study database consecutive patients were selected from seven centers (Catharina Hospital Eindhoven, Leiden University Medical Center, Rijnstate Hospital Arnhem, St. Elizabeth Hospital Tilburg, St. Franciscus Hospital Rotterdam, University Medical Center Utrecht, VU Medical Center Amsterdam). We selected these centers from all DUST centers because of their respective start date of inclusion and availability of the data at the time of patient selection for this study.

Additional inclusion criteria for this study were: admission between May 2009 and July 2011 with a CTP deficit in the region of the middle cerebral artery and an extended CTP acquisition. Exclusion criteria were technical failure of the extended CTP (incomplete scan series, excessive movement or artifacts).

General baseline patient characteristics were collected on admission: age, sex, prior history of cardiovascular risk factors obtained by anamnesis (stroke, hypertension, diabetes, hyperlipidemia, angina, myocardial infarction), NIHSS, time from stroke onset to admission CT scan and blood glucose $(\mathrm{mmol} / \mathrm{l})$. Determinants were selected on the basis of prevalence in stroke population and potential influence on the integrity of the BBB.

Imaging protocol

Non contrast brain CT (NCCT), CTP with extended acquisition, and CTA of the cervical and cerebral vessel were performed on admission before possible thrombolytic treatment was initiated. Multi-detector CT scanners used were Philips iCT 256-detector, Philips 64-detector, Philips 40-detector, Toshiba 320-detector and Toshiba 64-detector. CT protocols were adjusted to scanner type and contrast agent used.

The CTP involved successive gantry rotations in cine mode during intravenous administration of iodinated contrast material ( $40 \mathrm{ml} \mathrm{non-ionic} \mathrm{contrast} \mathrm{followed} \mathrm{by} 40 \mathrm{ml}$ of saline with a flow of $6 \mathrm{ml} / \mathrm{sec}$ ). Temporal sampling was obtained every $2 \mathrm{~s}$ for $50 \mathrm{~s}$ followed by six samples $30 \mathrm{~s}$ apart at 60, 90,120,150,180 and 210 s. Only the Philips scanners required a separate scan to change the cycle time and the two separate scans were fused prior to permeability measurements. The Toshiba 320-detector scanner had full brain coverage. The other CT scanners had a coverage of 40-65 $\mathrm{mm}$ (8-13 $5 \mathrm{~mm}$ slices) from at least the level of the basal ganglia to the lateral ventricles to be able to compare between patients.

Following CTP, a CTA was performed from aortic arch to the vertex with 50-70 $\mathrm{ml}$ non-ionic contrast. The CTA data was not used for this study. All data and imaging processing was done centrally in Utrecht University Medical Center.

Image post processing

Cerebral blood volume (CBV), cerebral blood flow (CBF), mean transit time (MTT) and time to peak (TTP) were automatically calculated from CTP data utilizing commercially available CTP software (Extended Brilliance workstation 4.5, Philips Healthcare). The internal carotid artery (if available in the scan range) or anterior cerebral artery was chosen as arterial input function [10]. The superior saggital sinus was used as venous output function. A MTT threshold of $145 \%$ compared to non-affected side was used to define the ischemic area. Within this area a CBV value of less then 
$2.0 \mathrm{ml} / 100 \mathrm{~g}$ was used to define the infarct core and a CBV of $2.0 \mathrm{ml} / 100 \mathrm{~g}$ or more to define the penumbra [5].

BBBP was calculated with a commercially available software based on the Patlak model (Intellispace Portal system, Philips Healthcare) [11]. The Patlak model involves the fitting of a regression line to observations of time-density curves for each pixel and for intravascular reference function. The slope of these lines represents a local blood-tobrain transfer constant and is an indicator of BBBP values (perfusion derived permeability surface area product (PS) in $\mathrm{ml} / \mathrm{min} / 100 \mathrm{~g}$ ). To minimize the influence of larger size vessels, pixels with a CBV $>9 \mathrm{ml} / 100 \mathrm{~g}$ were removed. Circular ROIs of identical size $\left(100 \mathrm{~mm}^{2}\right)$ were drawn in the center of the CTP threshold defined penumbra and infarct core and mirror-imaged regions of interest (ROIs) were automatically drawn in the contra-lateral hemisphere (Fig. 1). To correct for the potential influence of $\mathrm{CBF}$, extraction ratio $((\mathrm{BBBP} / \mathrm{CBF}) \times 100 \%)$ values were obtained in all four ROIs $[12,13]$. All measurements were done blinded to other study data.

\section{Image analysis}

On the admission NCCT, the presence of early CT signs (ECTS), defined as hypodensity with or without swelling,

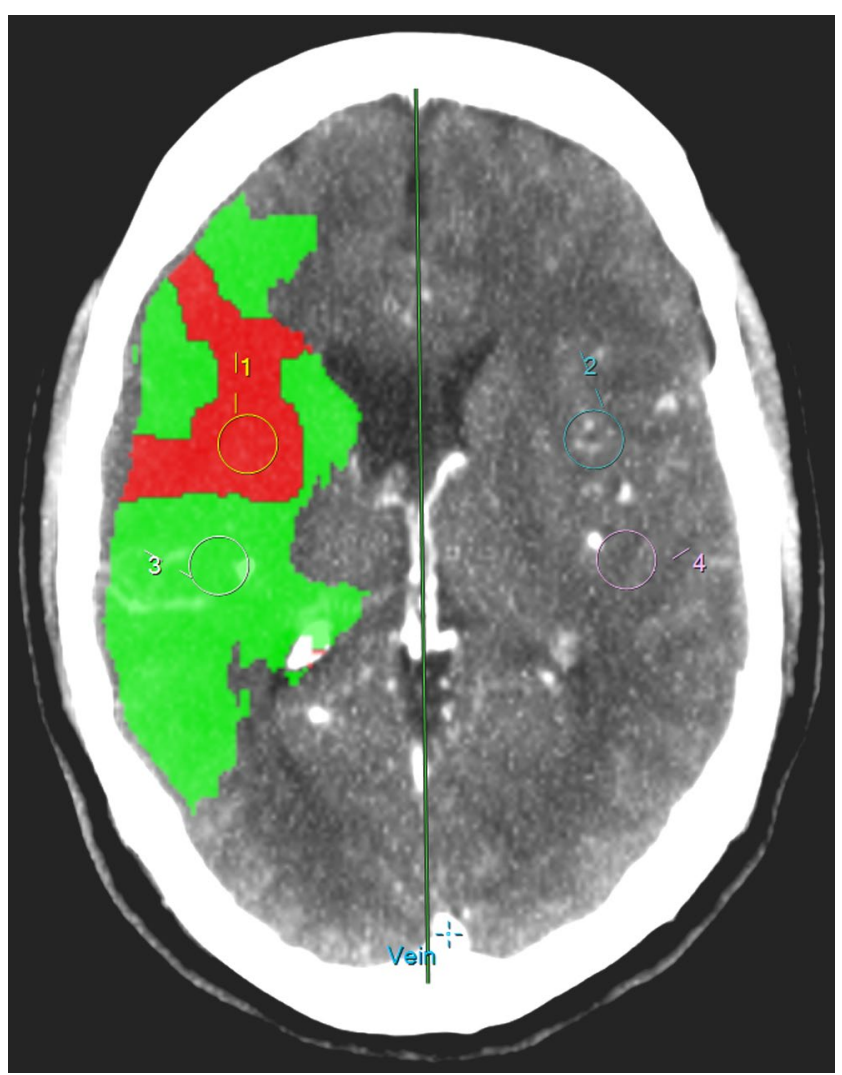

Fig. 1 Drawing of ROIs in infarct core and penumbra and presence of a dense vessel sign (DVS) were evaluated by one of three observers (I.C.vdS, B.K.V and J.W.D), all with at least 5 years of experience in stroke imaging. The side of symptoms was provided but observers were blinded to other clinical and imaging data. Consensus was reached for all discrepant findings.

Statistical analysis

Outcomes were BBBP in the penumbra- and infarct ROI and the contra-lateral hemisphere as well as BBBP ratios (ipsi-lateral/contra-lateral), and extraction ratio in both ROI's [14]. Variables referring to stroke severity were NIHSS (rearranged into quintiles), DVS, ECTS, and penumbra- and infarct size. Patient variables were age, sex, cardiovascular risk factors and glucose. Cardiovascular risk factors were dichotomized and considered positive if medical history included one of the following: hyperlipidemia, hypertension, diabetes, angina pectoris, myocardial infarction or atrial fibrillation. Prior to analysis outcome data were truncated to 5-95\% to adjust for outliers. Statistical computations were carried out using SPSS 19.0 (IBM corporation, NY, USA). The relation between the determinants and permeability values was quantified with regression analysis. In the multivariate analysis all variables were initially included and then sequentially removed with a probability-to-enter of 0.05 until only significant predictors remained (p 0.10).

\section{Results}

The inclusion criteria were met in 196 patients and 47 were subsequently excluded because of failed acquisition (22), motion artifacts (19) and beam hardening artifacts (6). Of the remaining 149 patients the stroke severity parameters and patient characteristics are summarized in Table 1 .

Outcome values are shown in Table 2. The median permeability value in the ipsi-lateral penumbra was 1.89 and in the infarct core 1.74; contra-lateral values were 1.71 and 1.59 respectively. The median extraction ratio in the infarct core was 17 and $10 \%$ for the penumbra, and respectively 4 and $3 \%$ on the contra-lateral side, with wide distribution. The median ratio of BBBP values between the ipsi-lateral and the mirrored contra-lateral area was 0.97 for the infarct core and 1.14 in the penumbra.

Univariate analysis (Table 3 ) showed significantly higher $\mathrm{BBBP}$ values in the infarct core $(\mathrm{B}=0.276)$ in the presence of ECTS but not in the penumbra or contra-lateral ROIs. ECTS $(B=18.7)$ and infarct size $(B=0.463)$ were also related to higher extraction ratio in the infarct core. Additionally, lower permeability values in penumbra $(B=-0.495)$ and infarct core $(B=-0.786)$ were found in female patients. All 
Table 1 Patient, stroke and imaging characteristics

\begin{tabular}{ll}
\hline Number of patients & 149 \\
Age in years, median (IQ range) & $69(59-80)$ \\
Sex, men \% & 54 \\
Patient characteristics: & \\
Cardiovascular risk factors, one or more of & 71 \\
the following \% & \\
Angina \% & 10 \\
Myocardial infarction \% & 13 \\
Atrial fibrillation \% & 13 \\
Diabetes \% & 16 \\
Hyperlipidemia \% & 30 \\
Hypertension \% & 57 \\
Glucose in mmol/1, median (IQ range) & $7.1(5.8-7.6)$ \\
Stroke severity: & $10(8,1-32)$ \\
NIHSS, mean (median, range) & $3.0(2.2,0.47-9)$ \\
Time from symptom onset to scan in hours, \\
mean (median, range) & 60 \\
0-3 h \% & 24 \\
3-4.5 h \% & 9 \\
4.5-6 h \% & 7 \\
6-9 h \% & \\
Imaging parameters: & 34 \\
Dense vessel sign (DVS) \% & 38 \\
Early CT signs (ECTS) \% & $21(6-40)$ \\
Penumbra size in cm ${ }^{2}$ median (IQ range) & $5(1-17)$ \\
Infarct size in cm ${ }^{2}$, median (IQ range) &
\end{tabular}

other parameters, including dense vessel sign, did not show significantly higher or lower BBBP values.

In multivariate analysis (Table 4) ECTS were related to higher $\mathrm{BBBP}$ values in the infarct core $(\mathrm{B}=0.710)$, higher ipsi- to contra-lateral $\mathrm{BBBP}$ ratios $(\mathrm{B}=0.326)$ and higher extraction ratios in the infarct core $(B=16.938)$. Females
Table 2 Median BBB Permeability measurements

\begin{tabular}{|c|c|c|c|}
\hline & $\begin{array}{l}\text { Ipsi-lateral } \\
\text { (IQ range) }\end{array}$ & $\begin{array}{l}\text { Contra-lateral } \\
\text { (IQ range) }\end{array}$ & $P$-value \\
\hline \multicolumn{4}{|c|}{ BBB Permeability (in $\mathrm{ml} / 100 \mathrm{gr} / \mathrm{min}$ ) } \\
\hline Penumbra & $1.89(1.23-2.79)$ & $1.71(1.15-2.73)$ & 0.3 \\
\hline Infarct core & $1.74(0.90-2.99)$ & $1.59(1.03-2.59)$ & 0.6 \\
\hline \multicolumn{4}{|c|}{ Extraction ratio (BBB Permeability/ CBF $* 100 \%$ ) } \\
\hline Penumbra & $10.1(5.65-16.76)$ & $3.2(2.16-6.18)$ & a \\
\hline Infarct core & $16.7(9.38-33.26)$ & $4.1(2.21-7.57)$ & a \\
\hline \multicolumn{4}{|c|}{ Ratio BBB Permeability ipsi-lateral/ contra-lateral } \\
\hline Penumbra & 1.1 & $(0.79-1.61)$ & N/A \\
\hline Infarct core & 1.0 & $(0.67-1.53)$ & N/A \\
\hline
\end{tabular}

showed lower BBBP values in penumbra and infarct core $(B=-0.446$ and -0.776 respectively) and lower extraction ratios in the infarct core $(B=-10.463)$. If a DVS is present the ipsi- to contra-lateral BBBP ratios in the infarct core were lower $(\mathrm{B}=-0.304)$.

\section{Discussion}

The most important finding in this study is that only ECTS are related to higher permeability values in the infarct core on admission, when calculated using the Patlak model. No significant differences were found between median BBBP values in the penumbra, infarct core and the unaffected contra-lateral hemisphere.

Median infarct BBBP values in our study fall in the range of previously published values $(0.34-3.5 \mathrm{ml} / 100 \mathrm{gr} / \mathrm{min})$ [15-18]. Median penumbra BBBP values have only been

Table 3 Univariate analysis of the relation between stroke severity, patient characteristics and BBB Permeability values (in penumbra and infarct core), and extraction ratio and BBB Permeability ratio (in infarct core)

\begin{tabular}{|c|c|c|c|c|c|c|c|c|}
\hline \multirow{2}{*}{$\begin{array}{l}\text { Outcome: } \\
\text { Predictor: }\end{array}$} & \multicolumn{2}{|c|}{$\begin{array}{l}\text { BBB Permeability } \\
\text { penumbra }\end{array}$} & \multicolumn{2}{|c|}{$\begin{array}{l}\text { BBB Permeability infarct } \\
\text { core }\end{array}$} & \multicolumn{2}{|c|}{ Extraction ratio infarct core } & \multicolumn{2}{|c|}{$\begin{array}{l}\text { BBB Permeability ratio } \\
\text { infarct core }\end{array}$} \\
\hline & $\mathrm{B}$ & $95 \% \mathrm{CI}$ & B & $95 \% \mathrm{CI}$ & B & $95 \% \mathrm{CI}$ & B & $95 \% \mathrm{CI}$ \\
\hline Age (in years) & -0.008 & $-0.026-0.010$ & -0.020 & $-0.042-0.003$ & -0.197 & $-0.633-0.238$ & -0.006 & $-0.018-0.006$ \\
\hline emale sex & $-0.495^{*}$ & $-0.958--0.032$ & $-0.786^{*}$ & $-1.386--0.186$ & -11.251 & $-22.540-0.038$ & -0.097 & $-0.428-0.235$ \\
\hline NIHSS $^{\mathrm{a}}$ & -0.043 & $-0.217-0.131$ & -0.007 & $-0.238-0.223$ & 2.200 & $-2.032-6.432$ & -0.081 & 0.042 \\
\hline Time to scan ${ }^{\mathrm{b}}$ & 0.008 & $-0.097-0.112$ & 0.049 & $-0.090-0.188$ & -0.260 & $-2.794-2.273$ & 0.013 & -0.087 \\
\hline $\begin{array}{l}\text { ardiovascular risk } \\
\text { actors }(\text { yes } / \text { no })^{c}\end{array}$ & 0.222 & $-0.280-0.725$ & -0.065 & $-0.726-0.596$ & 2.731 & $-9.550-15.012$ & 0.167 & $-0.189-0.523$ \\
\hline Glucose (mmol/l) & 0.064 & $-0.083-0.212$ & 0.012 & $-0.180-0.204$ & 2.247 & $-1.122-5.616$ & -0.019 & -0.085 \\
\hline Dense vessel sign (yes/no) & 0.134 & $-0.360-0.629$ & 0.374 & $-0.256-1.004$ & 7.213 & $-4.742-19.167$ & -0.188 & $-0.526-0.151$ \\
\hline Early CT signs (yes/no) & 0.232 & $-0.247-0.712$ & $0.819^{*}$ & $0.214-1.424$ & $18.709^{*}$ & $7.397-30.020$ & 0.276 & $-0.055-0.607$ \\
\hline Penumbra size $\left(\mathrm{cm}^{2}\right)$ & 0.003 & $-0.009-0.015$ & 0.010 & $-0.005-0.026$ & 0.228 & $-0.063-0.520$ & -0.005 & $-0.013-0.004$ \\
\hline Infarct size $\left(\mathrm{cm}^{2}\right)$ & -0.003 & $-0.020-0.013$ & 0.007 & $-0.014-0.029$ & $0.463 *$ & $0.062-0.864$ & -0.003 & $-0.014-0.009$ \\
\hline
\end{tabular}

In 5 categories: 1-3, 4-6, 7-12, 13-17 and 18 or higher

'In 4 categories: $0-3,3-4,54.5-6$ and $6-9 \mathrm{~h}$

${ }^{c}$ Any from: hyperlipidemia, hypertension, diabetes, angina pectoris, myocardial infarction or atrial fibrillation $* P<0.05$ 
published in one study with a value of $2.48 \mathrm{ml} / 100 \mathrm{gr} / \mathrm{min}$, which was higher than the value found in this study [19]. Unfortunately, we could not find any significant difference between values calculated in the different brain tissue types. Also, the ratio of permeability in the infarct core and the penumbra compared to the contra-lateral side was close to one, further that measured permeability values were similar in both hemispheres. This raises serious concerns about the applied technique. The finding that BBBP calculated with the Patlak model is not zero in the unaffected hemisphere suggests that a large level of noise influences the measurement of permeability in the used CTP acquisitions. The absence of differences between the different tissue types implies that the signal from the leaked contrast is not large enough to overcome the level of noise. Advanced noise filtering may be a solution to these problems [20]. The lack of signal will most likely be largest in an area of least flow, since hardly any contrast enters the tissue and can therefore not leak out. Our intention to overcome this issue by calculating the extraction ratio did result in more obvious differences between the tissue types, however with a very wide distribution.

In our multivariate analysis of factors describing stroke severity on BBBP only ECTS showed a positive relationship with higher BBBP values. Since ECTS are a sign of severe and often irreversible ischemia, our findings suggest that only severe ischemia induces measurable BBB breakdown within the first hours after acute ischemic stroke [21, 22]. The size of the ischemic deficit does not seem to influence BBBP values, as there is no significant relationship with infarct core or penumbra size. It still needs to be evaluated whether BBBP is a better predictor of HT in comparison to ECTS.

The lower ipsi-lateral to contra-lateral ratios of BBBP found in the infarct core when a dense vessel sign is present is difficult to explain as we expected higher- instead of lower values. A DVS has a high specificity and positive predictive value for diagnosing acute occlusion of the M1 segment of the MCA $[23,24]$. In some articles a DVS in the MCA is associated with more severe neurologic deficit at presentation, more extensive area of brain infarction, and worse neurologic outcome after thrombolysis [23-25]. In contrast, other studies found no relation between DVS and poor outcome [26-29]. A possible explanation for the lower values in this study is that hyperdense MCA sign (M1 segment) and MCA dot sign (M2 or higher) were grouped together although the prognosis of a M2 segment MCA occlusion was found to be better by Barber et al. 2001 [30]. Secondly, DVS is related to local vessel occlusion but this does not necessarily lead to tissue hypo-perfusion if distal collaterals provide sufficient perfusion.

Of the factors describing patient characteristics only female gender was associated with lower BBBP values. 
There are clear gender differences in acute ischemic stroke and estrogens and pro-inflammatory cytokine production during cerebral ischemia are thought to be related to these differences [31, 32]. The relationship between female sex and reduced disruption of the BBB has been found in several animal studies but so far could not be confirmed in human studies [19, 33-36]. Our study with human subjects does confirm that some relation between female sex and BBB integrity in acute ischemic stroke exists, but further studies are still needed to clarify this issue. In contrast to Bang et al. 2009, age and NIHSS were not related to significant differences in permeability values [6].

Besides the low signal-to-noise ratio of the BBBP measurements with the Patlak model from CTP data we acknowledge several other limitations to our study. First, we had to exclude nearly $25 \%$ of patients due to technical failure of the CTP. Compared to the numbers found in other studies our percentage is rather high. The technical failure was largely related to the extended acquisition necessary to calculate BBBP with the Patlak model [4, 17]. At present, not all $\mathrm{CT}$ vendors can change the cycle time from the initial two-second cycle time to the $30 \mathrm{~s}$ extended cycle time. This requires two separate perfusion scans which can cause difficulty in merging the two datasets for analysis. In addition, extending the acquisition time from 50 to $210 \mathrm{~s}$ also increases the risk of motion artefacts, which are especially difficult to correct in the z-direction. Second, we only used the Patlak model in this study which assumes unidirectional transfer without backflow and requires steady state contrast levels. This assumption is possibly not sufficiently accurate and technical improvement with other models (e.g. distributed parameter model) needs to be tested. The first results of such a model have been published recently [37]. Measurable BBBP values above the noise level are prerequisite to be able to predict possible hemorrhagic transformation. Third, No gold standard for the definition of infarct core and penumbra with CTP has been established so far. It is possible that when using other thresholds described in literature, the ROI's would have been different, resulting in other permeability values that might have correlated differently with BBBP. However, the definition of infarct core and penumbra we used has frequently been applied in literature and is the default setting in the post-processing software we used. This makes our results more reproducible for general stroke work-up when this software is used.

In conclusion, BBB permeability assessment with the Patlak model from CTP acquisition in stroke patients is technically challenging and limited by the current signal-tonoise ratio. In this study we found higher BBBP measurements when early ischemic changes on admission NCCT are present. This suggests that severe ischemic damage results in measurable BBBP alterations within the first hours after symptom onset.
Acknowledgements The DUST investigators are:

Academic Medical Center, Amsterdam, The Netherlands (Majoie CB, Roos YB); Catharina Hospital, Eindhoven, The Netherlands (Duijm LE, Keizer K); Erasmus Medical Center, Rotterdam, The Netherlands (van der Lugt A, Dippel DW); Gelre Hospitals, Apeldoorn, The Netherlands (Droogh - de Greve KE, Bienfait HP); Leiden University Medical Center, Leiden, The Netherlands (van Walderveen MA, Wermer MJ); Medical Center Haaglanden, The Hague, The Netherlands (Lycklama à Nijeholt GJ, Boiten J); Onze Lieve Vrouwe Gasthuis, Amsterdam, The Netherlands (Duyndam D, Kwa VI); Radboud University Nijmegen Medical Centre, Nijmegen, The Netherlands (Meijer FJ, van Dijk EJ); Rijnstate Hospital, Arnhem, The Netherlands (Kesselring FO, Hofmeijer J); St. Antonius Hospital, Nieuwegein, The Netherlands (Vos JA, Schonewille WJ); St. Elisabeth Hospital, Tilburg, The Netherlands (van Rooij WJ, de Kort PL); St. Franciscus Hospital, Rotterdam, The Netherlands (Pleiter CC, Bakker SL); VU Medical Center, Amsterdam, The Netherlands (Bot J, Visser MC); University Medical Center Utrecht, Utrecht, The Netherlands (Velthuis BK, van der Schaaf IC, Dankbaar JW, Mali WP, van Seeters T, Horsch AD, Niesten JM, Biessels GJ, Kappelle LJ, Luitse MJ, van der Graaf Y).

Sources of funding This study was supported by grants from the Netherlands Heart Foundation (grant numbers 2008 T034 and 2012 T061) and the Nuts Ohra Foundation (grant number 0903-012)

Ethical standards This study has been performed in accordance with the ethical standards laid down in the 1964 Declaration of Helsinki and its later amendments.

Declaration of conflict of interest BKV is a regular presenter for Philips

Open Access This article is distributed under the terms of the Creative Commons Attribution License which permits any use, distribution, and reproduction in any medium, provided the original author(s) and the source are credited.

\section{References}

1. Sandoval KE, Witt KA. Blood-brain barrier tight junction permeability and ischemic stroke. Neurobiol Dis. 2008;32(2):200-19.

2. Aviv RI, d'Esterre CD, Murphy BD, Hopyan JJ, Buck B, Mallia G, Li V, Zhang L, Symons SP, Lee TY. Hemorrhagic transformation of ischemic stroke: prediction with CT perfusion. Radiology. 2009;250(3):867-77.

3. Hom J, Dankbaar JW, Soares BP, Schneider T, Cheng SC, Bredno J, Lau BC, Smith W, Dillon WP, Wintermark M. Blood-brain barrier permeability assessed by perfusion ct predicts symptomatic hemorrhagic transformation and malignant edema in acute ischemic stroke. AJNR Am J Neuroradiol. 2011;32(1):41-8.

4. Hom J, Dankbaar JW, Schneider T, Cheng SC, Bredno J, Wintermark M. Optimal duration of acquisition for dynamic perfusion ct assessment of blood-brain barrier permeability using the patlak model. AJNR Am J Neuroradiol. 2009;30(7):1366-70.

5. Wintermark M, Flanders AE, Velthuis B, Meuli R, van Leeuwen M, Goldsher D, Pineda C, Serena J, van der Schaaf I, Waaijer A, Anderson J, Nesbit G, Gabriely I, Medina V, Quiles A, Pohlman S, Quist M, Schnyder P, Bogousslavsky J, Dillon WP, Pedraza S. Perfusion-CT assessment of infarct core and penumbra: receiver operating characteristic curve analysis in 130 patients suspected of acute hemispheric stroke. Stroke. 2006;37(4):979-85. 
6. Bang OY, Saver JL, Alger JR, Shah SH, Buck BH, Starkman S, Ovbiagele B, Liebeskind DS; UCLA MRI Permeability Investigators. Patterns and predictors of blood-brain barrier permeability derangements in acute ischemic stroke. Stroke. 2009;40(2):454-61.

7. Farrall AJ, Wardlaw JM. Blood-brain barrier: ageing and microvascular disease-systematic review and meta-analysis. Neurobiol Aging. 2009;30(3):337-52.

8. Starr JM, Wardlaw J, Ferguson K, MacLullich A, Deary IJ, Marshall I. Increased blood-brain barrier permeability in type II diabetes demonstrated by gadolinium magnetic resonance imaging. $\mathrm{J}$ Neurol Neurosurg Psychiatry. 2003;74(1):70-6.

9. van Seeters T, Biessels GJ, van der Schaaf IC, Dankbaar JW, Horsch AD, Luitse MJ, Niesten JM, Mali WP, Kappelle LJ, van der Graaf Y, Velthuis BK; DUST investigators. Prediction of outcome in patients with suspected acute ischaemic stroke with CT perfusion and CT angiography: the Dutch acute stroke trial (DUST) study protocol. BMC Neurol. 2014;14:37.

10. Niesten JM, van der Schaaf IC, Riordan AJ, de Jong HW, Mali WP, Velthuis BK. Optimisation of vascular input and output functions in CT-perfusion imaging using 256(or more)-slice multidetector CT. Eur Radiol. 2013;23(5):1242-9.

11. Patlak CS, Blasberg RG, Fenstermacher JD. Graphical evaluation of blood-to-brain transfer constants from multiple-time uptake data. J Cereb Blood Flow Metab. 1983;3(1):1-7.

12. Bisdas S, Hartel M, Cheong LH, Koh TS, Vogl TJ. Prediction of subsequent hemorrhage in acute ischemic stroke using permeability CT imaging and a distributed parameter tracer kinetic model. J Neuroradiol. 2007;34(2):101-8.

13. Dankbaar JW, Hom J, Schneider T, Cheng SC, Lau BC, van der Schaaf I, Virmani S, Pohlman S, Wintermark M. Age- and anatomy-related values of blood-brain barrier permeability measured by perfusion-CT in non-stroke patients. J Neuroradiol. 2009;36(4):219-27.

14. Dankbaar JW, Rijsdijk M, van der Schaaf IC, Velthuis BK, Wermer MJ, Rinkel GJ. Relationship between vasospasm, cerebral perfusion, and delayed cerebral ischemia after aneurysmal subarachnoid hemorrhage. Neuroradiology. 2009;51(12):813-9.

15. Thornhill RE, Chen S, Rammo W, Mikulis DJ, Kassner A. Contrast-enhanced MR imaging in acute ischemic stroke: T2* measures of blood-brain barrier permeability and their relationship to $\mathrm{T} 1$ estimates and hemorrhagic transformation. AJNR Am J Neuroradiol. 2010;31(6):1015-22.

16. Wu S, Thornhill RE, Chen S, Rammo W, Mikulis DJ, Kassner A. Relative recirculation: a fast, model-free surrogate for the measurement of blood-brain barrier permeability and the prediction of hemorrhagic transformation in acute ischemic stroke. Invest Radiol. 2009;44(10):662-8.

17. Vidarsson L, Thornhill RE, Liu F, Mikulis DJ, Kassner A. Quantitative permeability magnetic resonance imaging in acute ischemic stroke: how long do we need to scan? Magn Reson Imaging. 2009;27(9):1216-22.

18. Kassner A, Roberts TPL, Moran B, Silver FL, Mikulis DJ. Recombinant tissue plasminogen activator increases blood-brain barrier disruption in acute ischemic stroke: an MR imaging permeability study. AJNR Am J Neuroradiol. 2009;30(10):1864-9.

19. Dankbaar JW, Hom J, Schneider T, Cheng SC, Bredno J, Lau BC, van der Schaaf IC, Wintermark M. Dynamic perfusion-CT assessment of early changes in blood brain barrier permeability of acute ischaemic stroke patients. J Neuroradiol. 2011;38(3):161-6.

20. Mendrik AM, Vonken EJ, van Ginneken B, de Jong HW, Riordan A, van Seeters T, Smit EJ, Viergever MA, Prokop M. TIPS bilateral noise reduction in 4D CT perfusion scans produces high-quality cerebral blood flow maps. Phys Med Biol. 2011;56(13):3857-72.

21. von Kummer R, Bourquain H, Bastianello S, Bozzao L, Manelfe C, Meier D, Hacke W. Early prediction of irreversible brain damage after ischemic stroke at CT. Radiology. 2001;219(1):95-100.
22. Somford DM, Marks MP, Thijs VN, Tong DC. Association of early CT abnormalities, infarct size, and apparent diffusion coefficient reduction in acute ischemic stroke. AJNR Am J Neuroradiol. 2004;25(6):933-8.

23. Bastianello S, Pierallini A, Colonnese C, Brughitta G, Angeloni U, Antonelli M, Fantozzi LM, Fieschi C, Bozzao L. Hyperdense middle cerebral artery CT sign. Comparison with angiography in the acute phase of ischemic supratentorial infarction. Neuroradiology. 1991;33(3):207-11.

24. Mattle HP, Arnold M, Georgiadis D, Baumann C, Nedeltchev K, Benninger D, Remonda L, von Büdingen C, Diana A, Pangalu A, Schroth G, Baumgartner RW. Comparison of intraarterial and intravenous thrombolysis for ischemic stroke with hyperdense middle cerebral artery sign. Stroke. 2008;39(2):379-83.

25. Agarwal P, Kumar S, Hariharan S, Eshkar N, Verro P, Cohen B, Sen S. Hyperdense middle cerebral artery sign: can it be used to select intra-arterial versus intravenous thrombolysis in acute ischemic stroke? Cerebrovasc Dis. 2004;17(2-3):182-90.

26. Leys D, Pruvo JP, Godefroy O, Rondepierre P, Leclerc X. Prevalence and significance of hyperdense middle cerebral artery in acute stroke. Stroke. 1992;23(3):317-24.

27. Tomsick T, Brott T, Barsan W, Broderick J, Haley EC, Spilker J, Khoury J. Prognostic value of the hyperdense middle cerebral artery sign and stroke scale score before ultraearly thrombolytic therapy. AJNR Am J Neuroradiol. 1996;17(1):79-85.

28. Rauch RA, Bazan C 3rd, Larsson EM, Jinkins JR. Hyperdense middle cerebral arteries identified on $\mathrm{CT}$ as a false sign of vascular occlusion. AJNR Am J Neuroradiol. 1993;14(3):669-73.

29. Roberts HC, Dillon WP, Furlan AJ, Wechsler LR, Rowley HA, Fischbein NJ, Higashida RT, Kase C, Schulz GA, Lu Y, Firszt $\mathrm{CM}$. Computed tomographic findings in patients undergoing intraarterial thrombolysis for acute ischemic stroke due to middle cerebral artery occlusion: results from the PROACT II trial. Stroke. 2002;33(6):1557-65.

30. Barber PA, Demchuk AM, Hudon ME, Pexman JH, Hill MD, Buchan AM. Hyperdense sylvian fissure MCA "dot" sign: a CT marker of acute ischemia. Stroke. 2001;32(1):84-8.

31. Czlonkowska A, Ciesielska A, Gromadzka G, Kurkowska-Jastrzebska I. Gender differences in neurological disease: role of estrogens and cytokines. Endocrine. [Review]. 2006;29(2):243-56.

32. Madsen TE, Seigel TA, Mackenzie RS, Marcolini EG, Wira CR, Healy ME, Wright DW, Gentile NT. Gender differences in neurologic emergencies part I: a consensus summary and research agenda on cerebrovascular disease. Acad Emerg Med. 2014;21(12):1403-13.

33. Semenas E, Sharma HS, Nozari A, Basu S, Wiklund L. Neuroprotective effects of 17beta-estradiol after hypovolemic cardiac arrest in immature piglets: the role of nitric oxide and peroxidation. Shock. 2011;36(1):30-7.

34. Anishchenko TG, Brill GE, Romanova TP, Igosheva NB. Sex differences in adrenocortical sensitivity and resistance to cerebrovascular damage in rats under strong stress. Biull Eksp Biol Med. 1992;114(10):353-5.

35. Sirav B, Seyhan N. Blood-brain barrier disruption by continuous-wave radio frequency radiation. Electromagn Biol Med. 2009;28(2):215-22.

36. Oztas B, Akgul S, Seker FB. Gender difference in the influence of antioxidants on the blood-brain barrier permeability during pentylenetetrazol-induced seizures in hyperthermic rat pups. Biol Trace Elem Res. 2007;118(1):77-83.

37. Bennink E, Riordan AJ, Horsch AD, Dankbaar JW, Velthuis BK, de Jong HW. A fast nonlinear regression method for estimating permeability in CT perfusion imaging. J Cereb Blood Flow Metab. 2013;33(11):1743-51. 